\title{
Spectrum Analysis of Anode Rod Current in 300kA Aluminum Reduction Cells Based on Hilbert-Huang Transform
}

\author{
Shuiping Zeng ${ }^{1, a}$, Ruiqiang $\mathrm{Li}^{2, \mathrm{~b}}$ \\ ${ }^{1,2}$ Automation Department, North China University of Technology, Beijing, 100144, China \\ aemail:zshp@ncut.edu.cn, bemail:13810053161@139.com
}

Keywords: Aluminium Electrolysis; Anode Current; Spectrum Analysis; Hilbert-Huang Transform

\begin{abstract}
There are dozens of anodes work in aluminum electrolysis at the same time. Because of the particularity of anode work and the waving ability of the melt, different current flow through different anode. Spectral characteristics of the current signal are important parameters of internal status changes of aluminum reduction cell. Hilbert-Huang Transform is applied to analyze the spectrum of anode current signal. The procedure include Empirical Mode Decomposition, Hilbert transform, marginal spectrum calculation, and compare it to FFT algorithm. The results show that the frequencies of the aluminum electrolysis anode current signal are between $0.004 \mathrm{~Hz}$ and $0.005 \mathrm{~Hz}$. Finally, the technological characteristics of aluminum electrolysis shows the rationality of the analysis and further the feasibility of fault diagnosis of aluminum reduction cell.
\end{abstract}

\section{Introduction}

Aluminum electrolysis has the characteristics of non-linearity and strong corrosiveness. Electrolysis cell voltage and equidistant voltage drop of anode rod and their variations can reflect the status changes of electrolytic cell, so it is important to make an analysis for these signals. If anode current has problems during the process will become the cause of many problems like current imbalances which can cause the electrolytic cell to not work properly, and ultimately lead to lower electrolysis efficiency ${ }^{[1]}$. With people's research in anode current, the anode current in different electrolytic cell status have different characteristics in both time and frequency domain which helps us know the process of operation of electrolytic cell running normal or not.

For operation of electrolytic cell, some researchers have made spectrum analysis on the electrolysis cell voltage, and other researchers gathered the equidistant voltage drop of anode rod and based on the frequency spectrum of this signal analyzed the movement of the melt. Most of previous analysis are static signal processing, the method of stationary signal analysis was commonly used for it $^{[2,3,4]}$. Because of changing of aluminum reduction cell, the acquisition of signals is nonlinear and non-stationary so that signals' time varying information are not easily got by using FFT algorithm, other methods need choosing basis functions. The HHT algorithm to analyze the signal is based on the original signal so we don't need to select basis function before the signal transformation. HHT algorithm can process the signals which are nonlinear and non-stationary adaptively, and also can observe the variation of signal frequency ${ }^{[5,6]}$. This is very important to the dynamic analysis of the operation of electrolytic cell and the fault diagnosis, so HHT algorithm is adopted to be the analysis method in this paper.

\section{Data Analysis and Processing}

\subsection{Signals Processing Based on Hilbert-Huang Transform(HHT)}

A key part of HHT algorithm is EMD (Empirical Mode Decomposition). All non-stationary signals can be decomposed into a limited number of IMF (Intrinsic Mode Function) by EMD, and use Hilbert transform to solve each imfs' instantaneous frequency, then time-frequency Hilbert spectrums are acquired by means of EMD decomposition of original signal.

The termination condition is determined by the standard deviation $\left(S_{d}\right)$ of two continuous processing result. 


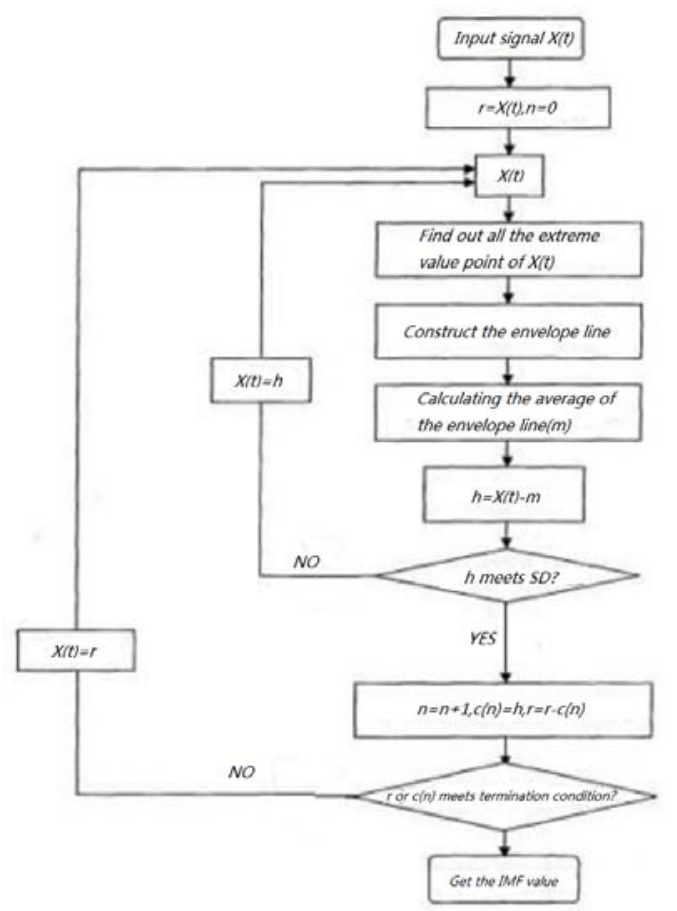

Fig. 1. EMD decomposition

$$
S_{d}=\sum_{t=0}^{T} \frac{\left|i m f_{(k-1)}(t)-i m f_{(k)}(t)\right|^{2}}{i m f_{(k-1)}(t)^{2}}
$$

In the formula (1), $S_{d}$ represents standard deviation and T represents signal's time span. We set $S_{d}$ equal to 0.2 .

We can get many imfs by means of EMD decomposition for original signal $x(t)$, symbolized by $_{1}(t), C_{2}(t) \ldots C_{n}(t)$. They contain the upper-to-lower frequency components of original signal, frequency components in every different band are different. Signal $\mathrm{x}(\mathrm{t})$ goes as follow.

$$
x(t)=\sum_{i=1}^{n} C_{i}+r_{n}
$$

In the formula $(2), \mathrm{C}_{\mathrm{i}}(\mathrm{t})$ represents component of $\mathrm{imf}$ and $\mathrm{r}_{\mathrm{n}}$ represents allowance.

\subsection{Signal Analysis of Equidistant Voltage Drop of Anode Rod}

Equidistant voltage drop of anode rod reflects current distribution in anode rod. A 300kA electrolytic cell in a Chinese factory have 20 anodes, and the signal of equidistant voltage drop of anode rod (about $20 \mathrm{~cm}$ long) is about $2-3 \mathrm{mV}$ during normal running. Whenever a failure happens, the signal of equidistant voltage drop of anode rod increases to $5-10 \mathrm{mV}$ quickly and returns to normal level after the failure. In this paper, based on a mathematical software MATLAB, we analyze this signal by HHT algorithm.

\subsubsection{Signal analysis of equidistant voltage drop of anode rod during normal running}

Because aluminum anode rod's conductivity changes with temperature, in order to facilitate the analysis, the original voltage data will be converted into voltage at the same temperature and do a removing mean processing on it. Figure 2 shows that the pre-processed signal of equidistant voltage drop of A1 anode rod during normal running.

Decompose this signal by empirical mode decomposition (EMD). Figure 3 shows that the results.

Once the condition is met when the signal is decomposed into imf11, stop the decomposition. Through HHT transform to each imf we can get instantaneous frequency. Figure 4 shows that the time-frequency decomposition results of imf3, imf5. 


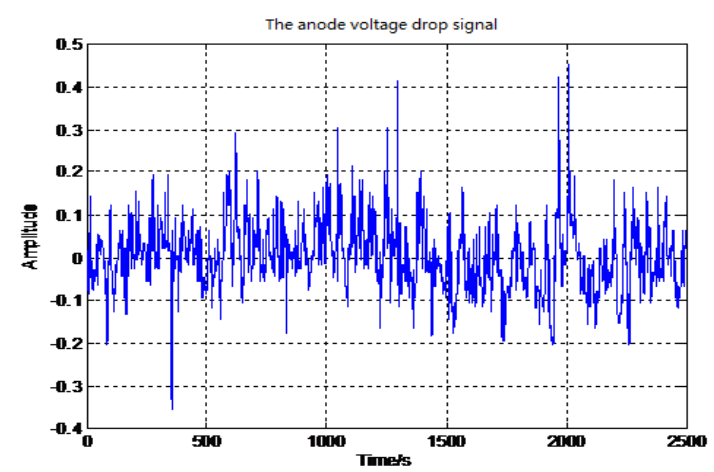

Fig. 2. The signal of equidistant voltage drop of anode rod A1

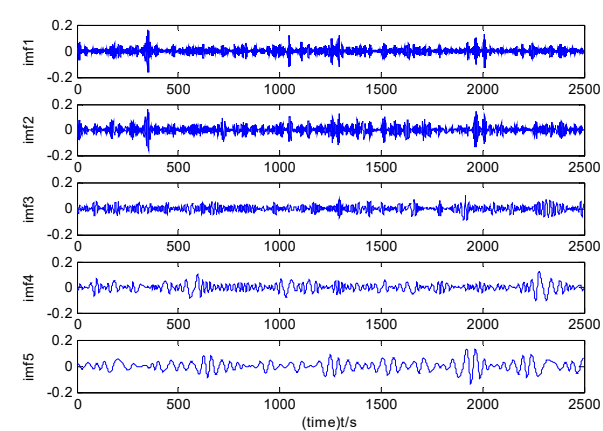

(a) imf1 $\sim \operatorname{imf5}$

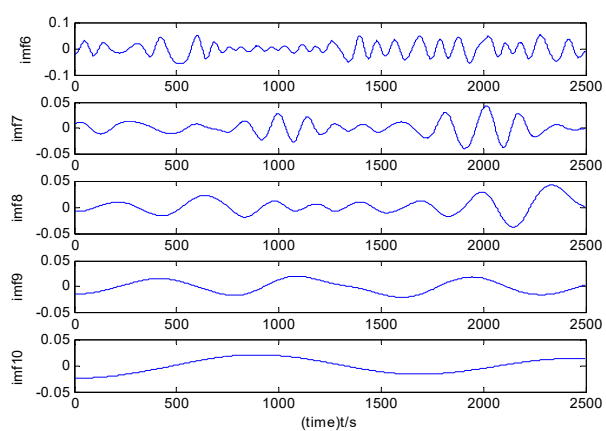

(b) imf6 $\sim \operatorname{imf10}$

Fig. 3. The results of imf1 imf10 by EMD

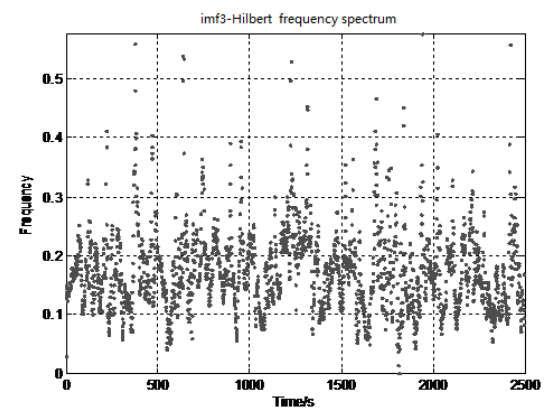

(a) $\operatorname{imf3}$

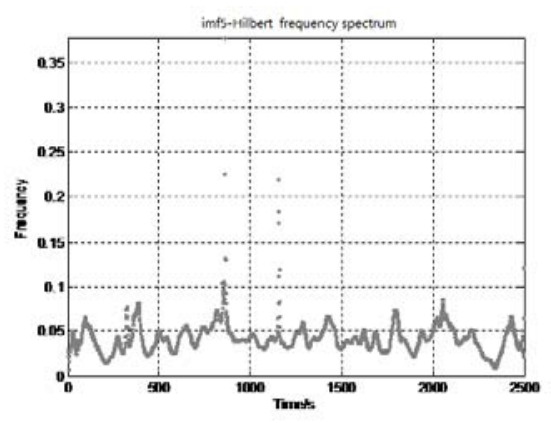

(b) imf5

Fig. 4. Hilbert frequency spectrum of imfs

As shown in the above figure, we can get the instantaneous frequency value of each imf by EMD decomposition to the signal of equidistant voltage drop of anode rod. We can find that Hilbert frequency spectrums of imfl imf 4 are very messy. The frequency of signals is high and between $0.1 \mathrm{~Hz}-0.5 \mathrm{~Hz}$, and contain a lot of frequency of noise and breakdown signal. Imf5 and later ones show us clearer frequency spectrums and the frequencies are concentrated over a low frequency range about $0 \mathrm{~Hz} \sim 0.03 \mathrm{~Hz}$. Imf7 and later ones' frequencies are very low frequency signals, so that they do not have much effect on aluminum electrolysis. Because EMD decomposition is based on original signal, the instantaneous frequency shown above can reflect real signal frequency component. We can observe the distributions and variations of different time signal frequencies, so that the instantaneous frequencies have physical meaning, it is useful for analysis of process property of aluminum electrolysis process.

Next, we make a marginal spectrum based on original signal. Compare the marginal spectrum to the result through FFT transform under the same conditions to find some laws. We can get the marginal spectrum (amplitude frequency spectrum) of original signal by integrating time $\mathrm{t}$ of $\mathrm{H}(\mathrm{w}, \mathrm{t})$, and compare it to the amplitude frequency spectrum through FFT method. Figure 5 shows that results.

Figure 5 shows that HHT method is more accurate, clearer than FFT in non-stationary signal processing. FFT may have false peak value. For instance, there is only one real peak value hidden 
Table 1. Extreme point of the marginal spectrum
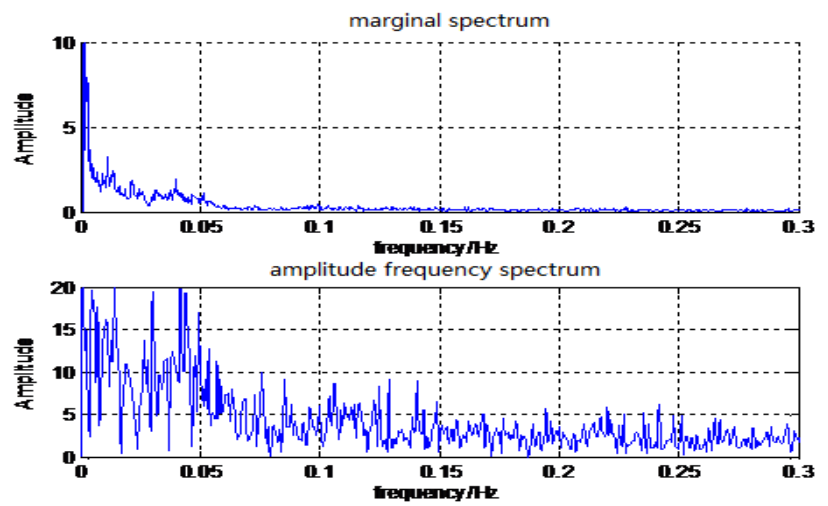

Fig. 5. Hilbert marginal spectrum and FFT amplitude frequency spectrum

\begin{tabular}{|c|c|c|c|}
\hline No & amplitude & frequency & period \\
\hline 0 & 15.91509 & 0.0012 & 833 \\
\hline 1 & 3.748723 & 0.004 & 250 \\
\hline 2 & 2.370483 & 0.0072 & 139 \\
\hline 3 & 2.31831 & 0.0092 & 109 \\
\hline 4 & 3.314641 & 0.0112 & 89 \\
\hline 5 & 2.306845 & 0.014 & 71 \\
\hline 6 & 1.176135 & 0.0168 & 60 \\
\hline 7 & 1.819272 & 0.022 & 45 \\
\hline 8 & 1.305164 & 0.0316 & 32 \\
\hline 9 & 1.192022 & 0.0416 & 24 \\
\hline 10 & 1.113492 & 0.0512 & 20 \\
\hline
\end{tabular}

from two adjacent peak value, but the possibility of two adjacent peak phenomenon is low during normal running. In figure 5, except the maximum peak, we can clearly observe direct current component of each characteristic value, and the main peak values of actual frequency of equidistant voltage drop of anode rod include 10 frequency range such as $0.004,0.007,0.0092,0.0112,0.014$, $0.0168,0.022,0.0316,0.0416,0.0512$. Corresponding signal periods are between 20-250 second.

The analysis of other anode has similar processes and results, but the position of peak value is different. This perfectly illustrates the different surroundings of anodes such as melt flow and fluctuation, furnace status and feeding. This problem will be discussed later on.

\subsubsection{Signal analysis of equidistant voltage drop of anode rod in abnormal condition}

We have the same process to analyze equidistant voltage drop of anode rod in abnormal condition, then the Hilbert frequency spectrum become more complex and changeable. Because of the similar analytic process and graph, we give the Hilbert marginal spectrum of signal and FFT amplitude frequency spectrum directly, as shown in figure 6.
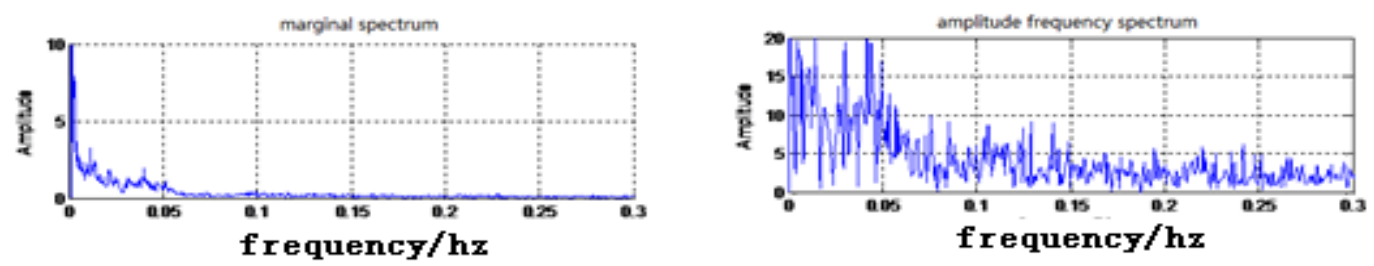

Fig. 6. Hilbert marginal spectrum and FFT amplitude frequency spectrum in abnormal condition

EMD decomposition and Hilbert spectrum analysis clearly reflect the composition of aluminum reduction cell signal and its dominant frequency range so that the study of signal analysis of aluminum electrolysis by HHT algorithm is very important. From the Hilbert marginal spectrum, we can observe the signal's dominant frequency range is between $0.004 \mathrm{~Hz} \sim 0.05 \mathrm{~Hz}$. We also can find that different levels of amplitude appeared at other frequency range such as $0.05 \mathrm{~Hz} \sim 0.2 \mathrm{~Hz}$, but the amplitude is lower. And in the same frequency range, there are so many interference of false frequencies in FFT amplitude-frequency figure. Literature ${ }^{[3]}$ consider the signal's dominant frequency range as $0.002 \mathrm{~Hz} \sim 0.04 \mathrm{~Hz}$, and literature ${ }^{[4]}$ consider the signal's dominant frequency range as $0.005 \mathrm{~Hz} \sim 0.05 \mathrm{~Hz}$. The results in this paper are similar to literatures, but the positions of characteristic points are different because of difference of type of cell and analysis method.

\section{Feature analysis and discussion of aluminum reduction cell}

Through the Hilbert frequency spectrum shown in figure 4 we can clearly observe the frequency components of anode current at different times are different. This change is closely related to various periodic operations of aluminum electrolysis during normal running. Especially for an individual anode, the period of feeding, aluminum liquid siphon, anode exchange and furnace variation are 
uncertain. The instantaneous frequency spectrum in this paper can reflect this uncertain periodic movement to a certain extent. Take median value imf5, for instance, the dominant frequency range is lower than $0.05 \mathrm{~Hz}$, but sometimes the frequency is higher than $0.05 \mathrm{~Hz}$ sometimes is lower than $0.02 \mathrm{~Hz}$ so that shows the major affecting factors of anode are changing in time. We can see from the figure 4 that this dynamic changes are regular. This problem will be discussed later on.

When the cell is running normally, there are many affecting factors of anode current. They are fluidity of liquid aluminum and electrolyte, waving ability of liquid aluminum and electrolyte, anode's gas bubble formation and escape, feeding of aluminum oxide and aluminum fluoride and outside interference of high frequency AC motor. Due to limited sampling frequency, we can only analyze low-frequency signal, and high-frequency signal cannot be analyzed. The interval generally 30 s to 90 s of feeding of aluminum oxide is controlled by control system which can analyze cell voltage real time, this is consistent with the signals after analysis such as $0.0112,0.014,0.0168,0.022$, 0.0316 .The interval generally $15 \mathrm{~s}$ to $90 \mathrm{~s}$ of node's bubble formation and escape is related to quality of electrolyte and surface quality of anode bottom, and the voltage is controlled by bubble's size, this is consistent with the signals after analysis such as $0.0416,0.0512$. The powerful signals after analysis such as $0.004,0.007,0.0092$ are affected by anode-cathode distance which is related to the fluidity of liquid aluminum and electrolyte and the waving ability of liquid aluminum and electrolyte.

\section{Conclusion}

According to reality of the factory, the equidistant voltage drop and temperature of anode rod are collected into database real time with $1 \mathrm{~Hz}$ sampling frequency. Based on Hilbert-Huang Transform algorithm, we have a research of the transformed signal of equidistant voltage drop of anode rod with spectrum analysis and get the real frequency component of signal. And it discusses the superiority of HHT compared with FFT. Finally, we get the main characteristics of signal of equidistant voltage drop of anode rod and corresponding characteristics of melt flow. The analysis indicates that the real frequency component of signal of equidistant voltage drop of anode rod includes:0.004, 0.007, 0.013, $0.03,0.04$. They are differently corresponding with electrolyte fluidity, electrolyte waving ability, feeding operation, anode's gas bubble formation and escape.

\section{Acknowledgements}

The research is sponsored by the National Natural Science Foundation of China (Project No. 51174007).

\section{References}

[1] Yexiang Liu, Jie Li, Modern Aluminum Electrolysis[M]. Beijing: Metallurgical Industry Press, 2008.

[2] Shuiping Zeng, Lin Cui, Jinhong Li. Diagnosis System for Alumina Reduction Based on BPNeural Network[J], JOURNAL OF COMPUTERS, 2012,7(4):929-933.

[3] Lei Ding, Shuiping Zeng, Zheng Zeng. 350kA Spectral Analysis of Cell Resistance Signals in 350kA Aluminum Reduction Cells[J]. Instrumentation and Measurement, 2005,24(12):68-77.

[4] Hesong Li. Diagnosis system of different status of aluminum reduction cells based on anode current fluctuation[J]. CIESC Journal,2011,62(6).

[5] Yi Shen, Zhiyuan Shen. A Review of the Nonlinear Nonstationary Adaptive Signal Processing Method-Hilbert-Huang Transform:Its Development and Applications[J].Techniques of Automation and Application, 2010,29(5):1-5.

[6] Lin Ye, Peng Liu. Combined Model Based on EMD-SVM for Short-term Wind Power Prediction[J]. Proceedings of the CSEE,2011, 31(31): 102-108. 